\title{
Evaluation of the hematologic system as a marker of subclinical inflammation in hyperemesis gravidarum: a case control study
}

\author{
Fatma Beyazitt ${ }^{1}$, Filiz Halici Öztürk² ${ }^{2}$ Eren Pek ${ }^{1}$, Mesut Abdülkerim Ünsal ${ }^{1}$ \\ ${ }^{1}$ Department of Obstetrics and Gynecology, Canakkale Onsekiz Mart University, Canakkale, Turkey \\ ${ }^{2}$ Department of Obstetrics and Gynecology, Canakkale State Hospital, Canakkale, Turkey
}

\begin{abstract}
Objectives: Current evidence suggests that subclinical inflammation plays a significant role in the development of hyperemesis gravidarum (HEG). Simple hematological markers, such as mean platelet volume (MPV), neutrophil-to-lymphocyte ratio (NLR), and platelet-to-lymphocyte ratio (PLR), have been shown to reflect inflammatory burden and disease activity in several disorders. This study aimed to determine the diagnostic value of these hematological parameters for HEG.

Material and methods: A total of $54 \mathrm{HEG}$ patients and 58 age- and gestational-age-matched control subjects were studied. NLR, MPV, PLR, platelet distribution width (PDW), and red cell distribution width (RDW) values in all patients were calculated and recorded from complete blood cell counts.

Results: For HEG patients, the median NLR was 3.2 (1.6-7.1), and the median PLR was 143.7 (78.1-334.6); for control subjects, the values were 2.1 (1.0-4.7) and 93.1 (47.3-194.7), respectively. Although both the NLR and PLR of HEG patients were found to be significantly higher than in the controls, no significant difference was found between the study groups in terms of MPV, RDW, or PDW. Correlation analysis revealed a significant correlation between NLR and CRP $(r=0.872, p<0.001)$.

Conclusions: Our results show that peripheral blood NLR and PLR values can reflect inflammatory burden in HEG patients and can be used as markers for HEG.
\end{abstract}

Key words: hyperemesis gravidarum, mean platelet volume, neutrophil-to-lymphocyte ratio, inflammation

\section{INTRODUCTION}

Hyperemesis gravidarum (HEG) is a medical condition of intractable vomiting during pregnancy; it has a multifactorial etiology and affects approximately $0.3-2 \%$ of all pregnancies [1]. In general, HEG occurs in the first trimester of pregnancy, but it can be seen as late as the twentieth week. Due to life-threatening complications such as weight loss, ketonuria, and electrolyte imbalance, it often requires hospitalization [2].

The exact pathophysiological basis of HEG is controversial and associated with multiple risk factors. HEG appears to begin with a complex interaction of various factors, including physiological, biological, and sociocultural factors. Hormonal changes (including high human chorionic gonadotropin [hCG] levels that cause transient hyperthyroidism), gastrointestinal dysfunction, hepatic dysfunction, metabolic derangements, lipid alterations, Helicobacter pylori infection, and genetic susceptibility have all been proposed to explain the underlying mechanisms of this potentially serious condition $[3,4]$. Although current data are inadequate to describe the role of inflammation in HEG pathogenesis, it seems likely that subclinical inflammation plays a causal role in HEG development $[5,6]$. The major pro-inflammatory cytokines, such as IL-6 and TNF-a, which are responsible for early inflammatory responses, are found to be elevated in HEG patients; this supports an inflammation-induced dysregulation of the biological system [6, 7].

Different hematological parameters have been used to evaluate the inflammatory status of distinct disease states 
in recent years $[8,9]$. Mean platelet volume (MPV), platelet distribution width (PDW), neutrophil-to-lymphocyte ratio (NLR), red cell distribution width (RDW) and platelet-to-lymphocyte ratio (PLR) are simple markers that have been shown to reflect inflammatory burden and disease activity in several disorders, including ulcerative colitis, spontaneous bacterial peritonitis, malignancies, and cardiovascular diseases $[10,11]$. With this background, the present study was undertaken to investigate the diagnostic value of MPV, PDW, NLR, RDW, and PLR in HEG patients and their correlation with other inflammatory markers as indices of subclinical inflammation.

\section{MATERIAL AND METHODS}

A total of 54 pregnant women with HEG, at 6-13 weeks gestation, and 58 pregnant controls without any complaints and matched for gestational age were recruited for this study. The inclusion criteria for HEG patients were as follows: persistent nausea and vomiting, loss of more than $5 \%$ of body weight, the presence of at least one positive ketonuria test in a random urine specimen, and a singleton pregnancy. Patients with a history of ovulation induction, eating disorders, multiple gestation, cigarette smoking, gastrointestinal disorders, thyroid disorders, or urinary tract infections were excluded from the study. The control group consisted of pregnant women without any complaints or chronic or inflammatory disorders, matched for gestational age with the study group. The study was approved by the ethics committee of Canakkale Onsekiz Mart University and was conducted in accordance with the guidelines of the Helsinki Declaration.

The following data were recorded for each patient: age, body-mass index (BMI), gestational age, gravidity, and parity. The gestational age of the women was determined by using the first date of the last menstrual period and confirmed by ultrasonography. BMI was calculated by dividing body weight by the square of height in meters.

All blood samples were collected without anticoagulant use from the antecubital vein after an overnight fast. Complete blood count (CBC), erythrocyte sedimentation rate (ESR), and C-reactive protein (CRP) values were also recorded for each patient. All CBC analyses were performed in the hematology laboratory of our hospital using the same Beckman Coulter (High Wycombe, UK) Gen-S automated analyser for all samples.

CBC parameters, including white blood cell (WBC) count, neutrophil count, lymphocyte count, hemoglobin $(\mathrm{Hb})$, platelet count (PIt), MPV, PDW, and RDW, were all derived from patients' files. The NLR was calculated from the differential count by dividing the absolute neutrophil count by the absolute lymphocyte count; the PLR was calculated by dividing the platelet count by the lymphocyte count.

The primary goal of the study was to define a clinical relationship between MPV, NLR, PDW, PLR and RDW values and the presence of HEG. The secondary goal was to evaluate whether there is a correlation between these hematologic indices and other conventional inflammatory markers.

\section{Statistical analysis}

Statistical Package for Social Sciences (Version 18, SPSS Inc., Chicago, IL, USA) for Windows was used to analyse the data. Continuous variables were tested for normality with the Kolmogorov-Smirnov test. All normally distributed data were compared using Student's t test. Data found to be non-normally distributed were compared using the Mann-Whitney $U$ test. Data are expressed as mean \pm standard deviation or median (min-max). Receiver operating characteristic (ROC) curve analysis was used to identify the optimal cut-off values of NLR and PLR that detect HEG with maximum sensitivity and specificity. Spearman correlation analysis used for evaluation of a possible correlation between distinct variables. $P$ values below 0.05 were considered to be statistically significant.

\section{RESULTS}

Fifty-four patients with HEG and 58 control subjects were enrolled in the study. No statistically significant differences were observed in age, BMI, or gestational age between the two groups. Clinical characteristics of the study groups are summarized in Table 1.

Table 1. Demographic features of the patients and controls

\begin{tabular}{|l|c|c|c|}
\hline & $\begin{array}{c}\text { Hyperemesis gravidarum } \\
(\mathbf{n = 5 4 )}\end{array}$ & $\begin{array}{c}\text { Control groups } \\
(\mathbf{n}=\mathbf{5 8})\end{array}$ & $\mathbf{p}$ \\
\hline Age (years) & $25(17-43)$ & $27(19-44)$ & $\mathrm{NS}^{* *}$ \\
\hline BMI $\left[\mathrm{kg} / \mathrm{m}^{2}\right]$ & $23.67 \pm 3.21$ & $24.22 \pm 3.78$ & $\mathrm{NS}^{*}$ \\
\hline Gestational age (weeks) & $9(7-13)$ & $9(7-12)$ & $\mathrm{NS}^{* *}$ \\
\hline Gravidity & $2(1-4)$ & $2(1-5)$ & $\mathrm{NS}^{* *}$ \\
\hline Parity & $1(0-3)$ & $1(0-4)$ & $\mathrm{NS}^{* *}$ \\
\hline
\end{tabular}

Notes: *Student's t test and **Mann-Whitney U test was applied. The data were presented either as median (min-max) or mean \pm standard deviation; NS — not significant; $\mathrm{BMI}$ — body mass index 


\begin{tabular}{|c|c|c|c|}
\hline & $\begin{array}{l}\text { Hyperemesis gravidarum } \\
\qquad(\mathrm{n}=54)\end{array}$ & $\begin{array}{l}\text { Control groups } \\
\quad(n=58)\end{array}$ & $\mathbf{p}$ \\
\hline Hemoglobin [g/dL] & $12.5(9.7-14.7)$ & $12.7(10.2-13.9)$ & $N S^{* *}$ \\
\hline $\mathrm{WBC}\left[/ \mathrm{mm}^{3} \times 10^{3}\right]$ & $8.6 \pm 1.8$ & $8.7 \pm 1.8$ & $\mathrm{NS}^{*}$ \\
\hline Platelet $\left[/ \mathrm{mm}^{3} \times 10^{3}\right]$ & $258.5(138.0-437.0)$ & $262.0(161.0-409.0)$ & $N S^{* *}$ \\
\hline MPV [fL] & $8.7(6.7-11.0)$ & $8.8(7.2-11.0)$ & $N S^{* *}$ \\
\hline PDW (\%) & $16.3(15.6-18.1)$ & $16.2(11.9-17.8)$ & $N S^{* *}$ \\
\hline RDW (\%) & $12.1 \pm 1.4$ & $12.6 \pm 1.7$ & $N S^{*}$ \\
\hline NLR & $3.2(1.6-7.1)$ & $2.1(1.0-4.7)$ & $<0.001^{* *}$ \\
\hline PLR & $143.7(78.1-334.6)$ & $93.1(47.3-194.7)$ & $<0.001^{* *}$ \\
\hline CRP [mg/L] & $5.9(0.2-31.0)$ & $4.2(0.5-57.8)$ & $N S^{* *}$ \\
\hline $\mathrm{ESR}[\mathrm{mm} / \mathrm{h}]$ & $13.5(2.0-47.0)$ & $16.0(2.0-44.0)$ & $N S^{* *}$ \\
\hline
\end{tabular}

Notes: *Student's t test and **Mann-Whitney U test was applied. The data were presented either as median (min-max) or mean \pm standard deviation; NS — not significant

For HEG patients, the median NLR was 3.2 (1.6-7.1), and the median PLR was 143.7 (78.1-334.6); for control subjects, the values were 2.1 (1.0-4.7) and 93.1 (47.3-194.7), respectively. NLR and PLR values of HEG patients were significantly higher than those of the controls $(p<0.001$ for both NLR and PLR). Mean MPV, PDW, and RDW values in HEG patients were not significantly different from the controls (Tab. 2). Although not found to be statistically significant, a pattern of increased CRP levels was seen in HEG patients. No significant difference was seen in ESR or WBC levels between study groups (Tab. 2).

ROC curve analysis suggested that the optimum NLR cut-off point for HEG was 2.38; the sensitivity, specificity, positive predictive value (PPV), and negative predictive value (NPV) were $70.6 \%, 79.6 \%, 78.8 \%$, and $71.7 \%$, respectively (AUC: 0,818 ). The optimum PLR cut-off point for HEG was 100.4; sensitivity, specificity, PPV, and NPV were $60.3 \%$, $94.4 \%, 92.1 \%$, and $68.9 \%$, respectively (AUC: 0,817 ).

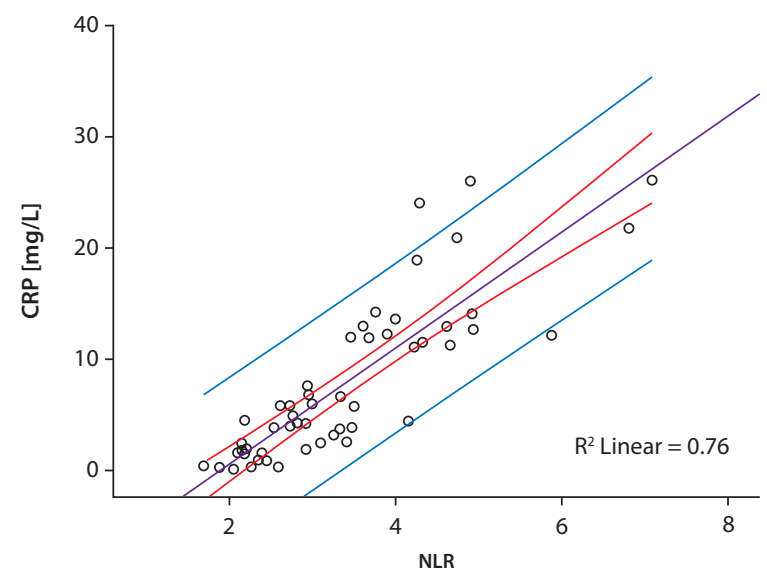

Figure 1. The correlation between neutrophil to lymphocyte ratio (NLR) and CRP. Lines representing the $95 \%$ confidence interval $(\mathrm{Cl})$ and the $95 \%$ prediction interval of the regression line
Table 3. Spearman correlation coefficients between NLR and PLR with other inflammation markers in HEG patients

\begin{tabular}{|r|c|c|c|}
\hline & CRP & ESR & WBC \\
\hline NLR & \multicolumn{1}{|c|}{} & \\
\hline$r_{s}$ & 0.872 & 0.263 & 0.476 \\
\hline$p$ & $<0.001$ & 0.065 & 0.071 \\
\hline PLR & & & \\
\hline$r_{s}$ & 0.299 & 0.188 & -0.116 \\
\hline$p$ & 0.058 & 0.192 & 0.402 \\
\hline
\end{tabular}

Although Spearman correlation analysis indicated a significant correlation between NLR and CRP $(r=0,872$, $p<0,001$; Figure 1), no correlation was found between ESR and WBC (Tab. 3)

\section{DISCUSSION}

The main aim of the present study was to evaluate the diagnostic value of MPV, NLR, RDW, PDW and PLR for HEG. We also sought a possible association between these markers and conventional inflammation markers such as CRP, ESR, and WBC. Our findings revealed that only NLR and PLR levels were higher in HEG patients than in controls. Also, only CRP levels were found to be correlated with NLR levels. NLR values were found to have high sensitivity, specificity, and predictive value in differentiating HEG patients from controls.

Though the role of inflammation in HEG pathophysiology is currently unclear, it is thought that oxidative stress, which accompanies subclinical inflammation, may play an important role [6, 12]. Kaplan et al. [7] evaluated serum cytokine levels in HEG patients as markers of inflammation; serum levels of TNF-a, which is a pleiotropic inflammatory cytokine produced by many cell types, were found to be elevated in HEG patients compared with healthy pregnant and 
nonpregnant women. In a recent study by Engin-Ustun et al. [5], serum vaspin and CRP levels were found to be elevated in HEG patients. A novel adipokine with insulin-sensitizing effects, vaspin acts as a pro-inflammatory cytokine, and elevated levels of vaspin in conjunction with elevated CRP levels support the idea of HEG as an inflammatory disorder [13]. Despite the well-known roles of these inflammatory markers in HEG pathogenesis, there are only scarce data in the literature describing the role of hematological markers in HEG pathogenesis. Therefore, this study was designed to evaluate the role of distinct hematological parameters, including NLR, MPV, RDW, PDW, and PLR in HEG patients, in conjunction with other conventional inflammatory markers.

The elevated NLR and PLR levels seen in our study could be the consequence of a physiological immune response of circulating leucocytes to HEG-associated physical stress, which results in amplification of neutrophils and a decrease in lymphocyte counts. In fact, this is the main reason that elevated NLR and PLR levels are found in peripheral blood analyses in all inflammatory disorders. In this context, NLR and PLR could be regarded as simple, inexpensive, and effective inflammation markers that have been linked with several inflammatory and neoplastic diseases [8]. Recent studies have shown that NLR and PLR values are strongly associated with the diagnosis and prognosis of ulcerative colitis, tubo-ovarian abscesses, familial Mediterranean fever, brucella arthritis, and colorectal cancer [8, 14-18]. Although PLR changes in HEG patients have not been studied previously, two recent studies demonstrated that NLR levels are increased and correlated in patients with HEG $[6,19]$. However, these studies did not compare the accuracy of the results with other hematological and inflammatory markers besides hsCRP.

Platelet size has been shown to be associated with platelet activation. Increases in platelet activation can be measured by MPV and PDW $[20,21]$. MPV is a measure of platelet size that shows platelet function and activation and can be measured by full blood count analysers as part of a routine CBC test cycle, with no additional cost [22]. It is one of the most widely used surrogate markers of platelet function and has been shown to reflect inflammatory burden and disease activity in several disease conditions, such as myocardial infarction, ulcerative colitis, familial Mediterranean fever, rheumatoid arthritis, acute ischemic stroke, and acute pancreatitis [23-25]. An extensive literature search revealed that no correlations have been studied in the analysis of MPV and PDW as hematological markers for HEG. Based on data showing that MPV and PDW values are associated with inflammation, we investigated if any alterations in MPV and PDW exist during the clinical course of HEG. We found no significant difference in MPV and PDW in HEG patients compared to controls, suggesting that there are other pathogenetic mechanisms in HEG than platelet activation.
RDW seems to be a predictor for a wide range of conditions, due to systemic factors such as inflammation and oxidative stress that alter erythrocyte homeostasis [26]. Inflammation might contribute to elevated levels of RDW not only by inhibiting the production of or response to erythropoietin, but also by impairing iron metabolism [27]. In our study, we found no significant difference in RDW levels between study groups. Moreover, no significant association between inflammation markers and RDW was observed.

There are some limitations of the present study that should be studied in future clinical investigations. The first major limitation of our study is the negligence of other well-demonstrated inflammatory markers such as TNF-a, IL-6 and IL-1 $\beta$. It would be worthwhile to study serum levels of these pro-inflammatory cytokines. Moreover, a correlation analysis between these cytokines and hematological markers could help to reveal the underpinnings of pathogenetic mechanisms that are difficult to discover otherwise. A second limitation is that, despite our findings, our results should be interpreted cautiously, because the relatively moderate sample size limited the power of the study. Finally, it would have been useful to evaluate the same HEG patients after clinical remission was achieved.

In conclusion, according to the results of the present study, although NLR and PLR can be considered efficient markers of disease, it is not certain whether high values of these markers are the result of a compensatory mechanism in response to HEG or if they reflect subclinical inflammation. A larger-scale, prospective clinical study should be conducted to further evaluate the diagnostic and prognostic significance of these hematological markers in HEG patients.

\section{REFERENCES}

1. Sandven I, Abdelnoor M, Nesheim Bl, et al. Helicobacter pylori infection and hyperemesis gravidarum: a systematic review and meta-analysis of case-control studies. Acta Obstet Gynecol Scand. 2009; 88(11): 1190 -1200, doi: 10.3109/00016340903284927, indexed in Pubmed: 19900137.

2. Ismail SK, Kenny L. Review on hyperemesis gravidarum. Best Pract Res Clin Gastroenterol. 2007; 21(5): 755-769, doi: 10.1016/j.bpg.2007.05.008, indexed in Pubmed: 17889806.

3. Kuşcu NK, Koyuncu F. Hyperemesis gravidarum: current concepts and management. Postgrad Med J. 2002; 78(916): 76-79, indexed in Pubmed: 11807187.

4. Goodwin TM. Hyperemesis gravidarum. Obstet Gynecol Clin North Am. 2008; 35(3): 401-17, viii, doi: 10.1016/j.ogc.2008.04.002, indexed in Pubmed: 18760227.

5. Engin-Ustun $Y$, Tonguç E, Var T, et al. Vaspin and C-reactive protein levels in hyperemesis gravidarum. Eur Rev Med Pharmacol Sci. 2013; 17(1): 138-140, indexed in Pubmed: 23329536.

6. Kurt RK, Güler A, Silfeler DB, et al. Relation of inflammatory markers with both presence and severity of hyperemesis gravidarum. Ginekol Pol. 2014; 85(8): 589-593, indexed in Pubmed: 25219138.

7. Kaplan PB, Gücer F, Sayin NC, et al. Maternal serum cytokine levels in women with hyperemesis gravidarum in the first trimester of pregnancy. Fertil Steril. 2003; 79(3): 498-502, indexed in Pubmed: 12620429.

8. Torun S, Tunc BD, Suvak B, et al. Assessment of neutrophil-lymphocyte ratio in ulcerative colitis: a promising marker in predicting disease severity. Clin Res Hepatol Gastroenterol. 2012; 36(5): 491-497, doi: 10.1016/j. clinre.2012.06.004, indexed in Pubmed: 22841412. 
9. Yildirim M, Turkyilmaz E, Avsar AF. Preoperative Neutrophil-to-Lymphocyte Ratio Has a Better Predictive Capacity in Diagnosing Tubo-Ovarian Abscess. Gynecol Obstet Invest. 2015; 80(4): 234-239, doi: 10.1159/ 1000371762, indexed in Pubmed: 25720652.

10. Suvak B, Torun S, Tas A, et al. Mean platelet volume is a useful indicator of systemic inflammation in cirrhotic patients with ascitic fluid infection. Ann Hepatol. 2013; 12(2): 294-300, indexed in Pubmed: 23396741.

11. Akbas EM, Demirtas L, Ozcicek A, et al. Association of epicardial adipose tissue, neutrophil-to-lymphocyte ratio and platelet-to-lymphocyte ratio with diabetic nephropathy. Int J Clin Exp Med. 2014; 7(7): 1794-1801, indexed in Pubmed: 25126182

12. Kuscu NK, Yildirim Y, Koyuncu F, et al. Interleukin-6 levels in hyperemesis gravidarum. Arch Gynecol Obstet. 2003; 269(1): 13-15, doi: 10.1007/s00404-002-0412-6, indexed in Pubmed: 14605817.

13. Wada J.Vaspin: a novel serpin with insulin-sensitizing effects. Expert Opin Investig Drugs. 2008; 17(3): 327-333, doi: 10.1517/13543784.17.3.327, indexed in Pubmed: 18321232.

14. Walsh SR, Cook EJ, Goulder F, et al. Neutrophil-lymphocyte ratio as a prognostic factor in colorectal cancer. J Surg Oncol. 2005; 91(3): 181-184, doi: 10.1002/jso.20329, indexed in Pubmed: 16118772.

15. Cho $\mathrm{S}, \mathrm{ChoH}, \mathrm{Nam} \mathrm{A}$, et al. Neutrophil-to-lymphocyte ratio as an adjunct to CA-125 for the diagnosis of endometriosis. Fertil Steril. 2008; 90(6): 20732079, doi: 10.1016/j.fertnstert.2008.03.061, indexed in Pubmed: 18555226.

16. Demir AK, Demirtas A, Kaya SU, et al. The relationship between the neutrophil-lymphocyte ratio and disease activity in patients with ulcerative colitis. Kaohsiung J Med Sci. 2015; 31(11): 585-590, doi: 10.1016/j. kjms.2015.10.001, indexed in Pubmed: 26678939.

17. Kim EY, Lee JW, Yoo HMo, et al. The Platelet-to-Lymphocyte Ratio Versus Neutrophil-to-Lymphocyte Ratio: Which is Better as a Prognostic Factor in Gastric Cancer? Ann Surg Oncol. 2015; 22(13): 4363-4370, doi: 10.1245/s10434-015-4518-z, indexed in Pubmed: 25805235.

18. Aktar F, Tekin R, Bektaş MS, et al. Diagnostic role of inflammatory markers in pediatric Brucella arthritis. Ital J Pediatr. 2016; 42: 3, doi: 10.1186/s13052-016-0211-5, indexed in Pubmed: 26753565.
19. Caglayan EK, Engin-Ustun $Y$, Gocmen AY, et al. Is there any relationship between serum sirtuin-1 level and neutrophil-lymphocyte ratio in hyperemesis gravidarum? J Perinat Med. 2016; 44(3): 315-320, doi: 10.1515/jpm-2015-0178, indexed in Pubmed: 26352076.

20. Huczek Z, Filipiak KJ, Kochman J, et al. Baseline platelet size is increased in patients with acute coronary syndromes developing early stent thrombosis and predicts future residual platelet reactivity. A case-control study. Thromb Res. 2010; 125(5):406-412, doi: 10.1016/j.thromres.2009.09.003, indexed in Pubmed: 19786298.

21. Ulucan Ş, Keser A, Kaya Z, et al. Association between PDW and Long Term Major Adverse Cardiac Events in Patients with Acute Coronary Syndrome. Heart Lung Circ. 2016; 25(1): 29-34, doi: 10.1016/j.hlc.2015.05.017, indexed in Pubmed: 26166174.

22. Sandhaus $L M$, Meyer $P$. How useful are $C B C$ and reticulocyte reports to clinicians? Am J Clin Pathol. 2002; 118(5): 787-793, doi: 10.1309/ /CQGG-HYOU-LRKL-GLMP, indexed in Pubmed: 12428801.

23. Bath PM, Butterworth RJ. Platelet size: measurement, physiology and vascular disease. Blood Coagul Fibrinolysis. 1996; 7(2): 157-161, indexed in Pubmed: 8735807.

24. Kisacik B, Tufan A, Kalyoncu U, et al. Mean platelet volume (MPV) as an inflammatory marker in ankylosing spondylitis and rheumatoid arthritis. Joint Bone Spine. 2008; 75(3): 291-294, doi: 10.1016/j. jbspin.2007.06.016, indexed in Pubmed: 18403245.

25. Sahin S, Senel S, Ataseven H, et al. Does mean platelet volume influence the attack or attack-free period in the patients with $\mathrm{Fa}$ milial Mediterranean fever? Platelets. 2013; 24(4): 320-323, doi: 10.3109/09537104.2012.697591, indexed in Pubmed: 22720903.

26. Özer S, Yılmaz R, Sönmezgöz E, et al. Simple markers for subclinical inflammation in patients with Familial Mediterranean Fever. Med Sci Monit. 2015; 21: 298-303, doi: 10.12659/MSM.892289, indexed in Pubmed: 25615955.

27. Weiss G, Goodnough LT. Anemia of chronic disease. N Engl J Med. 2005; 352(10): 1011-1023, doi: 10.1056/NEJMra041809, indexed in Pubmed: 15758012. 